\title{
Plasma intact fibroblast growth factor 23 levels in women with bulimia nervosa: A cross-sectional pilot study
}

\author{
Makoto Otani ${ }^{1,2^{*}}$, Yoshiyuki Takimoto ${ }^{1}$, Junko Moriya ${ }^{1}$, Kazuhiro Yoshiuchi ${ }^{1}$ and Akira Akabayashi $^{1}$
}

\begin{abstract}
Fibroblast growth factor (FGF) 23, a circulating 26-kDa peptide produced by osteogenic cells, is a novel phosphaturic factor. In our previous study, binge-eating/purging type anorexia nervosa (AN-BP) patients had elevated plasma intact FGF23 (iFGF23) levels, while restricting type (AN-R) patients had plasma iFGF23 levels similar to healthy controls. Although bulimia nervosa (BN) patients as well as some patients with AN-BP regularly engage in binge eating, there have been no studies regarding plasma iFGF23 levels in BN patients. Therefore, this study was performed to determine plasma iFGF23 concentrations in BN patients and healthy controls. The study population consisted of 13 female BN patients and 11 healthy female controls. Blood samples were collected from all subjects after overnight fasting. Plasma iFGF23 was measured using an ELISA kit in a cross-sectional manner. The two-tailed Mann-Whitney U-test was used to assess differences between BN patients and healthy controls. In addition, BN patients were divided into two groups based on questionnaire-reported binge eating frequency immediately prior to participation in this study: high frequency of binge eating (once a week or more; HF group; $n=8$ ) and low frequency of binge eating (less than once a week; LF group; $n=5$ ). Two-tailed Mann-Whitney $U$ test with Bonferroni's correction was performed after the Kruskal-Wallis test to assess differences between HF group, LF group, and healthy controls. Median (quartiles) plasma iFGF23 levels were greater in BN patients (35.5 [14.8-65.0] pg/ml) than in controls (3.8 [not detected-5.3] pg/ml; $\mathrm{p}=0.002$ ). In addition, median (quartiles) plasma iFGF23 levels were greater in the HF group $(62.3[44.4-73.4] \mathrm{pg} / \mathrm{ml})$ than in controls $(p<0.001)$ and in the LF group (12.9 [not detected-30.3] pg/ml; $p=0.011$ ), while there were no differences between the LF group and controls ( $p=0.441$ ). This is the first study to show that BN patients have elevated plasma iFGF23 levels. Moreover, this study showed that BN patients with a high frequency of binge eating have elevated plasma iFGF23 levels, while iFGF23 levels are similar to healthy controls in those with a low frequency of binge eating. Plasma iFGF23 level may be a suitable indicator of binge eating in $\mathrm{BN}$ patients.
\end{abstract}

Keywords: fibroblast growth factor 23 (FGF23), intact fibroblast growth factor 23 (iFGF23), eating disorders, bulimia nervosa (BN), binge eating, frequency of binge eating, dietary phosphate, plasma phosphate, 1,25-dihydroxyvitamin $\mathrm{D}\left(1,25-(\mathrm{OH})_{2} \mathrm{D}\right)$

\section{Findings}

Fibroblast growth factor (FGF) 23, a circulating 26-kDa peptide produced by osteogenic cells, is a novel phosphaturic factor, which is important for the regulation of inorganic phosphate homeostasis and for vitamin D metabolism [1]. FGF23 inhibits renal proximal tubule

\footnotetext{
* Correspondence: mootani-tky@umin.ac.jp

'Department of Stress Sciences and Psychosomatic Medicine, The University of Tokyo, 7-3-1 Hongo, Bunkyo-ku, Tokyo 113-8655, Japan

Full list of author information is available at the end of the article
}

phosphate reabsorption, increases renal phosphate excretion, and reduces serum phosphate without affecting serum calcium. FGF23 also strongly suppresses 1,25dihydroxyvitamin $\mathrm{D}\left(1,25-(\mathrm{OH})_{2} \mathrm{D}\right)$ production $[2,3]$.

Circulating FGF23 levels are regulated by serum phosphate $[4,5], 1,25-(\mathrm{OH})_{2} \mathrm{D}[3,6]$ and dietary phosphate [6]. Dietary phosphate plays an important role in FGF23 regulation, and dietary phosphate loading increases circulating FGF23 levels in healthy men in a matter of

\section{Biomed Central}

(c) 2011 Otani et al; licensee BioMed Central Ltd. This is an Open Access article distributed under the terms of the Creative Commons Attribution License (http://creativecommons.org/licenses/by/2.0), which permits unrestricted use, distribution, and reproduction in any medium, provided the original work is properly cited. 
days [7], even without changes in serum phosphate or 1,25- $(\mathrm{OH})_{2} \mathrm{D}$ levels [8].

Bulimia nervosa $(\mathrm{BN})$ is an eating disorder characterized by habitual binge eating, inappropriate compensatory behaviors, such as self-induced vomiting, a preoccupation with body weight, and excessive self-evaluation of weight and shape. For BN, the Diagnostic and Statistical Manual of Mental Disorders-Fourth Edition (DSM-IV) specifies that binging and compensatory behaviors must occur with a minimum average frequency and duration of at least twice a week for three months [9].

Our previous study showed that binge-eating/purging type anorexia nervosa (AN-BP) patients had elevated plasma intact FGF23 (iFGF23) levels, while restricting type (AN-R) patients had plasma iFGF23 levels similar to healthy controls [10]. Although BN patients as well as some patients with AN-BP regularly engage in binge eating, there have been no previous studies regarding plasma iFGF23 levels in BN patients. Therefore, the present study was performed to determine plasma iFGF23 concentrations in $\mathrm{BN}$ patients and healthy controls.

The study population consisted of 13 female outpatients of The University of Tokyo Hospital diagnosed with purging type BN according to DSM-IV by experienced clinicians, and 11 healthy female controls. All of the patients had binge eating episodes at least twice a week on average within the last three months, while the frequencies of binge eating episodes in some of the patients were small within the last two to four weeks just before the study because they were undergoing treatment. In addition, BN patients were divided into two groups on the basis of questionnaire-reported binge eating frequency just prior to participation in this study: patients with high frequency of binge eating (once a week or more; HF group; $n=8$ ) and patients with low frequency of binge eating (less than once a week; LF group; $n=5$ ). Physical comorbidity and medication directly affecting calcium and phosphate metabolism at the time of enrollment in this study were exclusionary criteria.

Blood samples were collected from all participants after overnight fasting. All BN patients completed a questionnaire about frequency of binge eating and vomiting for two weeks to one month just prior to participation in this study. Frequency of binge eating was rated on a six-point scale as follows: "hardly ever," "once a month," "once a week," "two or three times a week," "daily," and "two or three times a day." Frequency of vomiting was rated on a seven-point scale as follows: "never," "occasionally," "once a week," "two or three times a week," "daily," "two or three times a day," and "more than four times a day." The protocol was approved by the Institutional Ethics Committee of the University of Tokyo, and written informed consent was obtained from all subjects prior to enrollment in the study.
All blood samples were drawn into chilled tubes containing EDTA-2Na $(1 \mathrm{mg} / \mathrm{ml})$ and were then immediately centrifuged at $4{ }^{\circ} \mathrm{C}$. Plasma portions were stored at $-70^{\circ} \mathrm{C}$ prior to analysis. Plasma concentrations of iFGF23 were measured using an ELISA kit (Immutopics, San Clemente, CA) [10-12], with a sensitivity of $1.0 \mathrm{pg} / \mathrm{ml}$, intraassay variability of $<4.4 \%$, and interassay variability of $<6.5 \%$. All samples were analyzed in duplicate. Plasma $1,25-(\mathrm{OH})_{2} \mathrm{D}$ and 25-hydroxyvitamin D (25-OHD) concentrations were measured by RIA (SRL, Tokyo, Japan). Plasma calcium, phosphate, and intact parathyroid hormone (iPTH) concentrations were measured using standard laboratory methods (SRL).

The two-tailed Mann-Whitney U-test was used to assess the significance of differences between BN patients and healthy controls. The two-tailed Mann-Whitney U-test with Bonferroni's correction was performed after the Kruskal-Wallis test to assess the significance of differences between the HF group, LF group, and healthy controls. Values of $\mathrm{p}<0.05$ were considered significant, except that $\mathrm{p}<0.017$ was considered significant in the two-tailed Mann-Whitney U-test with Bonferroni's correction. Spearman's rank-correlation coefficients $(\rho)$ were used to assess the relationship between plasma iFGF23 levels and plasma phosphate or 1,25- $(\mathrm{OH})_{2} \mathrm{D}$ levels for BN patients. All statistical calculations were performed using SPSS for Windows version 10.0 (SPSS, Chicago, IL). All data are presented as the median, first quartile, and third quartile.

Clinical profiles and biochemical data are summarized in Table 1. Median (quartiles) plasma iFGF23 levels were significantly greater in all $\mathrm{BN}$ patients $(35.5[14.8-65.0] \mathrm{pg} /$ $\mathrm{ml}$ ) than in controls ( 3.8 [not detected -5.3 ] $\mathrm{pg} / \mathrm{ml} ; \mathrm{p}=$ 0.002). In addition, median (quartiles) plasma iFGF23 levels were significantly greater in the HF group (62.3 [44.4-73.4] $\mathrm{pg} / \mathrm{ml})$ than in healthy controls $(\mathrm{p}<0.001$; Figure 1) or the LF group (12.9 [not detected-30.3] pg/ml; $\mathrm{p}=0.011$; Figure 1), while there were no significant differences between the LF group and healthy controls ( $\mathrm{p}=$ 0.441; Figure 1). Binge eating behavior was significantly more frequent in the HF group than in the LF group $(\mathrm{p}=$ 0.002 ), while there were no differences in frequency of vomiting between the HF group and the LF group $(\mathrm{p}=0.222)$.

For all BN patients, iFGF23 levels were correlated with neither plasma phosphate levels $(\rho=0.160, p=0.602)$ nor plasma $1,25-(\mathrm{OH})_{2}$ D levels $(\rho=0.132, \mathrm{p}=0.667)$.

This is the first study to show that BN patients have elevated plasma iFGF23 levels. Moreover, the present study showed that BN patients with a high frequency of binge eating have elevated plasma iFGF23 levels, while the iFGF23 levels are similar to healthy controls in those with a low frequency of binge eating. These results suggest that plasma iFGF23 levels may be associated with binge eating frequency in $\mathrm{BN}$ patients. 
Table 1 Clinical profiles and biochemical data of women with bulimia nervosa and healthy controls

\begin{tabular}{|c|c|c|c|c|c|}
\hline & $\begin{array}{l}\text { BN patients with high frequency of } \\
\text { binge eating }(n=8)\end{array}$ & $\begin{array}{l}\text { BN patients with low frequency of } \\
\text { binge eating }(n=5)\end{array}$ & $\begin{array}{l}\text { Controls } \\
(\mathrm{n}=11)\end{array}$ & $\begin{array}{l}\text { Normal } \\
\text { values }\end{array}$ & $\mathrm{p}$ \\
\hline Body Mass Index $\left(\mathrm{kg} / \mathrm{m}^{2}\right)$ & $19.9(18.4-22.0)$ & $18.6(18.2-18.6)$ & $\begin{array}{l}19.7(19.5- \\
21.8)\end{array}$ & & 0.103 \\
\hline Age (years) & $28(25-28)$ & $25(23-27)$ & $27(21-29)$ & & 0.792 \\
\hline $\begin{array}{l}\text { age at the time of disease } \\
\text { onset (years) }\end{array}$ & $19(18-21)$ & $19(18-20)$ & & & \\
\hline disease duration (years) & $5.8(4.0-9.8)$ & $6.0(3.5-9.0)$ & & & \\
\hline frequency of binge eating & 2-3/week $(2-3 /$ week-1/day)* & $1 /$ month (1/month-1/month) & & & \\
\hline frequency of vomiting & 2-3/week (occasionally-1/day) & occasionally (occasionally-occasionally) & & & \\
\hline uncorrected Ca (mg/dl) & $8.8(8.7-9.3)$ & $9.4(9.2-9.7)$ & $8.9(8.7-9.1)$ & $8.5-10.2$ & 0.204 \\
\hline$P(\mathrm{mg} / \mathrm{dl})$ & $3.6(2.9-4.1)$ & $3.5(2.9-3.6)$ & $2.9(2.5-3.2)$ & $2.4-4.3$ & 0.133 \\
\hline intact PTH $(\mathrm{pg} / \mathrm{ml})$ & $62(43-69)$ & $54(45-55)$ & $38(33-45)$ & $10-65$ & 0.073 \\
\hline $1,25-(\mathrm{OH})_{2} \mathrm{D}(\mathrm{pg} / \mathrm{ml})$ & $39.4(17.1-55.6)$ & $38.2(37.7-42.9)$ & $\begin{array}{l}39.7(20.9- \\
47.5)\end{array}$ & $20.0-60.0$ & 0.837 \\
\hline $25-\mathrm{OHD}(\mathrm{ng} / \mathrm{ml})$ & $15(13-16)$ & $18(17-19)$ & $20(15-21)$ & $7-41$ & 0.186 \\
\hline
\end{tabular}

All values are shown as the median (first quartile-third quartile). The p-values in the rightmost column were calculated using the Kruskal-Wallis test.

${ }^{*} \mathrm{p}<0.05$ vs. BN patients with low frequency of binge eating (two-tailed Mann-Whitney U-test).

BN; bulimia nervosa. PTH; parathyroid hormone. 1,25-(OH) 2 D; 1,25-dihydroxyvitamin D. 25-OHD; 25-hydroxyvitamin D.

Gwirtsman et al. [13] showed that frequent vomiting increased serum amylase levels in BN patients. The serum amylase level is an established indicator of vomiting behavior. However, there are no established indicators of binge eating behavior. The establishment of an indicator of binge eating in $\mathrm{BN}$ patients would be therapeutically useful. Plasma iFGF23 level may be a suitable indicator of binge eating behavior in $\mathrm{BN}$ patients.

During binge eating, $\mathrm{BN}$ patients eat a large quantity of food at once, including foods such as chocolates, cakes, snacks, and sweet buns, which generally contain moderate to large amounts of phosphate. Thus, binge eating in BN patients may be regarded as dietary phosphate loading. It is speculated that dietary phosphate loading with binge

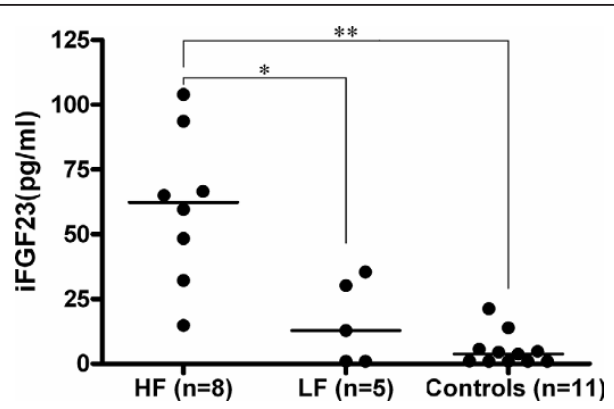

Figure 1 Dot plots of plasma intact fibroblast growth factor 23 (iFGF23) levels in bulimia nervosa (BN) patients with a high frequency of binge eating (HF group), BN patients with a low frequency of binge eating (LF group), and healthy controls. The graphs show median values (bars). The two-tailed Mann-Whitney Utest with Bonferroni's correction was used to assess the significance of differences among groups. A value of $p<0.017$ was considered statistically significant. ${ }^{*} p<0.017$ vs. LF group. ${ }^{* *} p<0.001$ vs. controls. eating may increase plasma iFGF23 levels in BN patients. The present study showed that there were no significant differences in plasma calcium, phosphate, iPTH, 1,25$(\mathrm{OH})_{2} \mathrm{D}$, or 25-OHD levels between the three groups. These results were consistent with the speculation.

The present study had three limitations. First, the number of BN patients was small. Second, the volumes of binge eating and purging in the $\mathrm{BN}$ patients prior to participation in the study were not available. Third, dietary phosphate intake prior to participation in the study was not assessed in BN patients. We were therefore unable to completely assess the relationships between binge eating behavior, dietary phosphate intake, and plasma iFGF23 levels. In future studies, dietary phosphate intake and the volume of binge eating prior to participation in the study should be determined in addition to plasma iFGF23, 1,25- $(\mathrm{OH})_{2} \mathrm{D}$, and 25-OHD levels.

This preliminary study showed that $\mathrm{BN}$ patients have elevated plasma iFGF23 levels, and that BN patients with a high frequency of binge eating have elevated plasma iFGF23 levels, while iFGF23 levels are similar to healthy controls in those with a low frequency of binge eating. Plasma iFGF23 level may be a suitable indicator of binge eating in $\mathrm{BN}$ patients.

\section{Acknowledgements and Funding}

Funding for this study was provided by Research Grant No. 14A-10 for Nervous and Mental Disorders from the Ministry of Health, Labor, and Welfare (MHLW) of Japan. The MHLW had had no further role in study design, in the collection, analysis and interpretation of data, in the writing of the report, or in the decision to submit the paper for publication.

\section{Author details}

'Department of Stress Sciences and Psychosomatic Medicine, The University of Tokyo, 7-3-1 Hongo, Bunkyo-ku, Tokyo 113-8655, Japan. ${ }^{2}$ Department of 


\section{Authors' contributions}

MO designed the study, analyzed the data, performed the statistical analysis, interpreted the results, and drafted the manuscript. JM collected the data. YT, KY, and AA helped analyze the data, interpret the results, and draft the manuscript. All authors have read and approved the final manuscript.

\section{Competing interests}

The authors declare that they have no competing interest.

Received: 18 January 2011 Accepted: 17 June 2011

Published: 17 June 2011

\section{References}

1. Quarles LD: FGF23, PHEX, and MEPE regulation of phosphate homeostasis and skeletal mineralization. Am J Physiol Endocrinol Metab 2003, 285:E1-E9.

2. Shimada T, Hasegawa H, Yamazaki Y, Muto T, Hino R, Takeuchi Y, Fujita T, Nakahara K, Fukumoto S, Yamashita T: FGF-23 is a potent regulator of vitamin D metabolism and phosphate homeostasis. I Bone Miner Res 2004, 9:29-435.

3. Shimada T, Yamazaki Y, Takahashi M, Hasegawa H, Urakawa I, Oshima T, Ono K, Takitani M, Tomizuka K, Fujita T, Fukumoto S, Yamashita T: Vitamin $D$ receptor-independent FGF23 actions in regulating phosphate and vitamin D metabolism. Am J Physiol Renal Physiol 2005, 289:F1088-F1095.

4. Weber TJ, Liu S, Indridason OS, Quarles LD: Serum FGF23 levels in normal and disordered phosphorus homeostasis. J Bone Miner Res 2003, 18:1227-1234.

5. Imanishi Y, Inaba M, Nakatsuka K, Nagasue K, Okuno S, Yoshihara A, Miura M, Miyauchi A, Kobayashi K, Miki T, Shoji T, Ishimura E, Nishizawa Y: FGF-23 in patients with end-stage renal disease on hemodialysis. Kidney Int 2004, 65:1943-1946.

6. Saito H, Maeda A, Ohtomo S, Hirata M, Kusano K, Kato S, Ogata E, Segawa H, Miyamoto K, Fukushima N: Circulating FGF-23 is regulated by 1a,25-dihydroxyvitamin $D_{3}$ and phosphorus in vivo. $J$ Biol Chem 2005, 280:2543-2549

7. Burnett SM, Gunawardene SC, Bringhurst FR, Jüppner $\mathrm{H}$, Lee $\mathrm{H}$, Finkelstein JS: Regulation of C-terminal and intact FGF-23 by dietary phosphate in men and women. J Bone Miner Res 2006, 21:1187-1196.

8. Ferrari SL, Bonjour JP, Rizzoli R: Fibroblast growth factor-23 relationship to dietary phosphate and renal phosphate handling in healthy young men. J Clin Endocrinol Metab 2005, 90:1519-1524.

9. American Psychiatric Association: Diagnostic and statistical manual of mental disorders. Washington $\mathrm{DC}_{i} 41994$

10. Otani M, Takimoto Y, Moriya J, Yoshiuchi K, Akabayashi A: Plasma intact fibroblast growth factor 23 levels in women with anorexia nervosa. Biopsychosoc Med 2008, 2(10):1-4.

11. Tebben PJ, Kalli K, Cliby WA, Hartmann LC, Grande JP, Singh RJ, Kumar R: Elevated fibroblast growth factor 23 in women with malignant ovarian tumors. Mayo Clin Proc 2005, 80:745-751.

12. Park SE, Cho MA, Kim SH, Rhee Y, Kang ES, Ahn CW, Cha BS, Lee EJ, Kim KR, Lee HC, Lim SK: The adaptation and relationship of FGF-23 to change in mineral metabolism in Grave's disease. Clin Endcrinol 2007, 66:854-858.

13. Gwirtsman HE, Kaye WH, George DT, Carosella NW, Greene RC, Jimerson DC: Hyperamylasemia and its relationship to binge-purge episodes: development of a clinically relevant laboratory test. $J$ Clin Psychiatry 1989, 50:196-204.

doi:10.1186/1751-0759-5-7

Cite this article as: Otani et al:: Plasma intact fibroblast growth factor 23 levels in women with bulimia nervosa: A cross-sectional pilot study. BioPsychoSocial Medicine 2011 5:7.

\section{Submit your next manuscript to BioMed Central and take full advantage of:}

- Convenient online submission

- Thorough peer review

- No space constraints or color figure charges

- Immediate publication on acceptance

- Inclusion in PubMed, CAS, Scopus and Google Scholar

- Research which is freely available for redistribution

Submit your manuscript at www.biomedcentral.com/submit
C Biomed Central 